\title{
Cognitive skills and literacy performance of Chinese adolescents with and without dyslexia
}

\author{
Kevin K. H. Chung - Connie S. -H. Ho • \\ David W. Chan $\cdot$ Suk-Man Tsang $\cdot$ Suk-Han Lee
}

Published online: 2 April 2010

(C) The Author(s) 2010. This article is published with open access at Springerlink.com

\begin{abstract}
The present study sought to identify cognitive abilities that might distinguish Hong Kong Chinese adolescents with dyslexia and to assess how these abilities were associated with Chinese word reading, word dictation, and reading comprehension. The cognitive skills of interest were morphological awareness, visual-orthographic knowledge, rapid naming, and verbal working memory. A total of 90 junior secondary school students, 30 dyslexic, 30 chronological age controls, and 30 reading level controls was tested on a range of cognitive and literacy tasks. Dyslexic students were less competent than the control students in all cognitive and literacy measures. The regression analyses also showed that verbal working memory, rapid naming, morphological awareness, and visual-orthographic knowledge were significantly associated with literacy performance. Findings underscore the importance of these cognitive skills for Chinese literacy acquisition. Overall, this study highlights the persistent difficulties of Chinese dyslexic adolescents who seem to have multiple causes for reading and spelling difficulties.
\end{abstract}

Keywords Adolescence Chinese language $\cdot$ Developmental dyslexia $\cdot$ Reading and spelling difficulties

\footnotetext{
K. K. H. Chung ( $ه)$ e-mail: kevin@ied.edu.hk

C. S. -H.Ho

The University of Hong Kong, Pokfulam, Hong Kong, China

D. W. Chan

The Chinese University of Hong Kong, Sha Tin, Hong Kong, China

S.-M. Tsang · S.-H. Lee

Education Bureau, Hong Kong SAR Government, Hong Kong, China
}

Social and Cognitive Neuroscience Unit, Department of Special Education and Counselling, The Hong Kong Institute of Education, 10 Lo Ping Road, Tai Po, NT, Hong Kong, China 


\section{Introduction}

Developmental dyslexia or reading disability is viewed as a disorder characterised by difficulties in learning to read and spell despite normal intelligence and in the absence of sensory impairment, brain damage, or environmental deprivation (Catts \& Kamhi, 2005; McBride-Chang, 1995). Dyslexia is now accepted as a life-span disorder. However, as is often the case in development, trajectories of reading and spelling achievement are not homogeneous and the manifestations of the cognitive deficits can change across the life span in dyslexic readers. Extensive work has focused on young children with dyslexia but little research has examined the reading-related cognitive skills underlying reading and writing problems in adolescent readers, particularly for non-alphabetic languages. Until recently, few studies have documented the progress of children with dyslexia (Bruck, 1990, 1992; Wolf \& Katzir-Cohen, 2001). Those that have done so reported a slow rate of reading and spelling development with poor levels of literacy into young adulthood (Bruck, 1992; Maughan \& Hagell, 1996; Shaywitz et al., 1999). The present study is to expand this work by investigating the development of metalinguistic skills associated with literacy abilities in Chinese adolescents with dyslexia during the transition from childhood to adolescence. Given that multiple deficits in a variety of cognitive skills were found in children, we drew from previous work on the cognitive skills associated with reading and writing ability, namely visual-orthographic knowledge, morphological awareness, rapid naming, and verbal short-term memory in alphabetic and non-alphabetic languages (e.g. de Jong \& van der Leij, 2003; Ho, Chan, Lee, Tsang, \& Luan, 2004; Ho, Chan, Tsang, \& Lee, 2002; Shu, McBrideChang, Wu, \& Liu, 2006). A weakness in any one of these four cognitive skills could contribute to literacy difficulties. Given the known correlations among these skills, it was critical to examine how these variables might distinguish adolescents with and without dyslexia and how they might be associated with word reading, word spelling (or word dictation), and reading comprehension. We briefly review the literature on the cognitive deficits in dyslexic individuals of different languages, and the characteristics of Chinese language. We then review the four reading-related cognitive skills highlighting why they may be particularly interesting and important to consider for Chinese adolescents.

Cognitive deficits in dyslexic readers of alphabetic languages

Extensive investigation across different languages has demonstrated the existence of a core phonological deficit in the reading and writing difficulties of dyslexic adolescents, especially in English speaking-readers (Bruck, 1990, 1992; Lehtola \& Lehto, 2000; Shaywitz et al., 1999). This cognitive deficit characterizes most dyslexic readers in adolescence and persists into their adulthood (Bruck, 1992; Felton, Naylor, \& Wood, 1990). Also important, and perhaps a separate issue, is a deficiency in name retrieval processes, measured by a rapid automatized naming (RAN) procedure (Wolf \& Bowers, 1999). This deficit in rapid naming which is related to the retrieval of phonological codes from memory (Wagner, Torgesen, Laughon, Simmons, \& Rashotte, 1993) or phonological encoding with lexical 
access (Wagner \& Torgesen, 1987) is thought to reflect the automatization of language subprocesses. In a number of studies, evidence was found that dyslexic individuals are slow in digit and letter naming speed and continue to have difficulty with rapid naming during adolescence (Felton et al., 1990; Korhonen, 1995). Apart from these deficits, they have additional difficulties in visual-orthographic processing (Corcos \& Willows, 1993) and morphological awareness (Carlisle, 1995; Leong, 1999). These difficulties are likely to affect readers' ability to encode printed words, to manipulate the word structures and to analyze the meaning of morphologically complex words. Moreover, adolescents with dyslexia have shortterm verbal memory (Torgesen et al., 1987; Wijsman et al., 2000) and long-term working memory difficulties (Hulme, 1981) that cause problems in creating word representations in memory and retrieving them from memory. Other studies reported that the manifestations of cognitive deficits can change over the life span (Bruck, 1990; Nation \& Snowling, 1998). With increasing literacy experience, the type and severity of cognitive deficiencies can be modified by the availability of compensatory resources, for example, semantic knowledge (Snowling, 2000), use of context (Nation \& Snowling, 1998), oral language, and rich vocabulary (Bruck, 1990) to provide some degree of compensation for reading and spelling difficulties. The preceding review indicates that the cognitive deficits causing literacy difficulties of adolescent dyslexia may not manifest themselves to the same extent as in childhood dyslexia.

Many of the studies have been restricted to English-speaking dyslexic adolescents. Current research evidence on individuals with a history of dyslexia has identified several underlying cognitive deficits in these readers namely, rapid naming, phonological and morphological awareness, visual-orthographic knowledge, and verbal working memory (e.g., Shaywitz et al., 1999; Shaywitz \& Shaywitz, 2005). There seem to be multiple causes for literacy difficulties of dyslexic adolescent individuals. It is understood that the characteristics of different languages could contribute differently to the types of cognitive deficits experienced by dyslexic readers. This then leads to the question of whether or to what extent readers with dyslexia in childhood are differentially affected by various cognitive deficits associated with reading and spelling difficulties in non-alphabetic languages, particularly for Chinese. Before discussing the developmental dyslexia in readers of Chinese, the main characteristics of the Chinese orthography will be reviewed.

\section{Characteristics of Chinese orthography}

Chinese is sometimes described as being a morphosyllabic writing system as each basic graphic unit of Chinese is the character which is associated with a morpheme (meaning unit) and represents a syllable of spoken Chinese (DeFrancis, 1989; Mattingly, 1984). About $80 \%$ of characters are ideophonetic compounds that are constructed from a meaning component called the semantic radical and a pronunciation component called the phonetic radical (Kang, 1993). An example is 燈 /dang1/, lamp, 火/fo2/, fire, is the semantic radical which gives a cue to the meaning of the character (as one needed fire to light an oil lamp in the olden days), 
and 登 /dang1/, climb is the phonetic radical which gives a cue to the pronunciation of the compound character. The numbers of semantic and phonetic radicals are approximately 200 and 800, respectively (Shu, Chen, Anderson, Wu, \& Xuan, 2003). Even though the majority of Chinese characters contain a phonetic radical, the information therein often provides an unreliable cue to its pronunciation. Compared to the phonetic radicals, the semantic radicals give more reliable cues and are directly linked to meaning (Shu et al., 2003). However, the association between component radicals and the sound or the meaning of the whole character is neither entirely transparent nor completely reliable since different degrees of semantic and phonological regularity are found in characters (Shu \& Anderson, 1997; Shu, Anderson, \& Wu, 2000). This makes intuitive sense given that for the majority of characters the relationship between orthography and phonology is rather arbitrary.

Apart from the functional regularity mentioned above, the positional regularity of radicals that is the core of a character's orthographic structure has an important role in its processing (Shu \& Anderson, 1997). Radicals usually inhabit certain positions in a character. Most semantic radicals occupy the left or top position within left-right (洗, /sai/, wash) or top-bottom (草, /cou2/, grass) structure. Feldman and Siok (1999) estimated about $75 \%$ of these radicals are on the left of a character. Similar to the regularity of letter sequences in English, the positions of all the semantic and phonetic radicals in a Chinese character may be a focus in determining the lexicality of the orthographic structure. If all radicals are in their legal positions, that character may either be a real character or a pseudocharacter. However, if the radicals are in their illegal positions, the character is a noncharacter. Also, Feldman and Siok (1997) suggested that the position of radicals may be confounded with function. Given the complex rules of positional and functional regularities, they are seldom taught in schools or addressed in textbooks explicitly. Thus the knowledge of radical position or character structure is learned through copying and memorization and this in turn does not help readers to learn new characters and to remember more characters efficiently.

In Chinese, the majority of words are multisyllabic with about two-thirds being bisyllabic (Taylor \& Taylor, 1995). Most Chinese words are built and compounded from two or more morphemes. Many words sharing the same morpheme such as 柳樹, /lau5 syu6/, willow, 松樹, /cung4 syu6/, pine, 棕櫚樹, /zung1 leoi3 syu6/, palm with the morpheme 樹, /syu6/, tree, are semantically related because the semantic radical of a Chinese character often provides some indication of its meaning. Also, there are a relatively large number of syllables that share the same sounds or homophones due to the limited number of syllables. Many syllables may have five or more homophones which provide different meaning (Packard, 2000; Zhou, Zhuang, \& Yu, 2002). For example, 身, /sai1/, body, 新, /sai1/, new, and 伸, /sai1/, extend. Therefore when learning to read it is helpful to be able to distinguish among these syllables with identical sounds and to figure out which character maps onto which in multiple speech syllables that sound identical. Moreover, knowing the compound of the word formation or morphological structural awareness is particularly important for learning Chinese vocabulary. An understanding of the morphosyllabic characteristic of Chinese orthography and of the semantic transparency of words is necessary in order to discover the nature of cognitive profile of Chinese speaking-readers with dyslexia. 
Cognitive deficits experienced by Chinese dyslexic readers

Existing research evidence on Chinese dyslexic readers has identified several underlying multiple cognitive deficits in these children including weakness of visual-orthographic skills, rapid naming, morphological awareness, verbal memory, and phonological awareness (Ho et al., 2002, 2004; Shu et al., 2006). Recent studies examined the different subtypes of dyslexic readers that exist among Chinese children who displayed the most dominant type of cognitive deficits, these being rapid naming, visual-orthographic processing, and morphological awareness (Ho et al., 2002, 2004; Shu et al., 2006). However, phonological awareness deficits, which were the less common subtype, could be of secondary importance due to the fact that the way Hong Kong Chinese readers learn to read (Ho, Chan, Chung, Lee, \& Tsang, 2007; Ho et al., 2004). Unlike readers in China and Taiwan, who learn to read Chinese with phonemic coding system (Pinyin) or an onset-rime system (Zhu-Yin-Fu-Hao) as an aid, Hong Kong Chinese readers learn to read characters in a more holistic manner by using a look-and-say approach. In this approach, students are taught to learn Chinese characters holistically including their visual configurations and pronunciation by rote. Thus, teachers often employ a drilling approach where Chinese characters are presented, named and read repetitively (Chan, Ho, Tsang, Lee, \& Chung, 2006). Therefore, phonemic awareness itself might not be useful in discriminating readers with dyslexia from non-impaired readers in Hong Kong Chinese sample. Given that phonological skills may not play an important role for learning to read Chinese, phonological awareness is not included in the present study. Because our interest in the present study was in identifying potential markers of reading and word spelling difficulties, the four cognitive areas of visualorthographic skills, rapid naming, morphological awareness, and verbal working memory are discussed below.

Visual-orthographic knowledge distinguishes children with dyslexia from those without dyslexia across a variety of alphabetic and non-alphabetic orthographies (Ho et al., 2002; Hultquist, 1997; Wolf \& Bowers, 1999). In Chinese, visualorthographic knowledge refers to children's awareness of conventional rules in structuring Chinese characters and their ability to identify or distinguish real Chinese characters from a pool of pseudocharacters, noncharacters, and visual symbols. Because of the great number of visually complicated Chinese characters, visual-orthographic processing at a basic level may be particularly important in learning to read Chinese. Visual-orthographic deficit was also demonstrated as one of the causes of reading failures with some children. Ho et al. (2002, 2004) also examined visual-orthographic processing of Chinese children with dyslexia in Hong Kong and found that orthographic deficit was one of the prominent problems faced by the Chinese children with dyslexia. Owing to the lack of reliable graphemephoneme correspondence of the Chinese characters, the knowledge about orthographic regularities of character structure and radicals may also be important for children learning to read Chinese. Given the complex rules of positional and functional regularities, Chinese dyslexic children tend to have difficulty in acquiring visual-orthographic knowledge and so may not be able to develop a strong orthographic representation of words in their mind, thus contributing to failures in 
Chinese. The present study, therefore, included a measure of visual-orthographic knowledge for distinguishing readers with and without reading disability.

Morphological awareness is another skill that has been found to be important for reading acquisition across languages. Recent research has shown that morphological awareness has a strong influence on reading processes, more particularly reading problems (Ben-Dror et al., 1995; Casalis \& Louis-Alexandre, 2000). Recently, Shu et al. (2006) reported that morphological awareness was one of the best discriminators for children with and without dyslexia. In Chinese, morphological awareness is conceptualized as the ability to distinguish meanings among morpheme homophones or as the ability to manipulate and access morphemes in words with two or more morphemes. Deficits in morphological awareness may well be especially prominent in Chinese language with its rich morphology. Another property of the Chinese script that differs significantly from alphabetic scripts is the extent of homophony. There are a relatively large number of syllables that share the same sounds or homophones due to the limited number of syllables. Many syllables may have more than one homophone which provide different meanings (Packard, 2000; Zhou et al., 2002). Therefore when learning to read, it is helpful to be able to distinguish among these syllables with identical sounds and to figure out which character maps onto which in multiple speech syllables that sound identical. Moreover, knowing the compound of the word formation or morphological structural awareness is particularly important for learning Chinese vocabulary. Seemingly, morphological ability could serve to distinguish different degrees of reading disabilities in Chinese (Shu et al., 2006), so sensitivity to morphological structures of language may be taken as significant factor influencing Chinese reading. The measures of morphological awareness were included for the present study.

Rapid automatized naming (RAN) is also an important general processing skill in learning to read for a variety of scripts (Ho \& Lai, 1999: Wimmer, Mayringer, \& Landerl, 2000). Ho and Lai found that Chinese children with dyslexia named digits, colors, pictures, and character words more slowly than did typically achieving children and suggested that these children appeared to have some generalized deficits in the speed of access to the lexicon or the ability to efficiently access lexical information that hindered their reading development. Subsequent cognitive profiling studies in Hong Kong reported that individual children with dyslexia exhibited multiple cognitive deficits, the dominant type of profile deficit being in rapid naming (Ho et al., 2002, 2004). Thus the RAN task contributes uniquely to reading deficits in some poor Chinese readers. Moreover, as noted in a study by Manis, Seidenberg, and Doi (1999), Chinese character recognition is relatively "arbitrary" and a rapid naming measure may tap into the ability to learn arbitrary links between print and sound. Because both Chinese character recognition and a rapid naming task involve the automatic mapping of arbitrary language and print information, Chinese may be a writing system that is particularly strongly associated with a rapid naming task which becomes uniquely related to children's reading performance. Therefore, the rapid naming is one of the strongest correlates of Chinese word reading (Leong, Tse, Loh, \& Hau, 2008; McBride-Chang et al., 2008), and among the most dominant types of cognitive deficits found in Hong Kong Chinese 
individuals with dyslexia so far (Ho et al., 2002, 2004). The measure of rapid naming was included in the present study as well.

Verbal working memory has also been found to be associated with reading acquisition (Hu \& Catts, 1998). It has been suggested that the function of verbal short-term memory is to support the long-term learning of the phonological structure of the language (Baddeley, Gathercole, \& Papagno, 1998). Consequently, individuals with inadequate verbal memory skills may experience difficulties in learning the phonological structures of novel words. Furthermore, Fowler (1991) argued that poor readers tended to experience significant difficulties in encoding, rehearsing, storing and retrieving speech stimuli from memory. Indeed, there is substantial evidence that poor readers perform less well on measures of verbal memory (Siegel \& Ryan, 1988; Swanson, 1994). For instance, Zhang, Zhang, Chang, and Zhou (1998) found that Chinese children with dyslexia performed worse than did the typically achieving children in verbal memory tasks such as the tasks of digit memory and other complex memory measures. In other studies, Stevenson, Stigler, Lucker, Hsu, and Kitamura (1982) demonstrated that poor Chinese readers made more errors in verbal memory and memory for words than did the typically achieving children. From the findings, Chinese dyslexic children appear to have relative weakness in keeping sounds and verbal information in their short-term memory, adversely affecting their development of stable graphic-sound associations and their acquisition of visual-orthographic knowledge and these problems in turn impair typical reading development. Ho and colleagues suggested that verbal memory deficits appear to be one of the prominent problems faced by Chinese children with dyslexia (Ho, Law, \& Ng, 2000; Ho \& Lai, 1999). Although developmental consequences of impairments of verbal working memory contribute to reading difficulties during early childhood years, the mechanisms underlying the link between the two domains are not as yet fully understood in Chinese adolescent readers with developmental dyslexia. Therefore, we included a measure of verbal working memory in the present study.

All of the above research concerning developmental dyslexia shows that a variety of cognitive skills that distinguish children with dyslexia are likely to contribute significantly to literacy difficulties. Although the manifestations of multiple cognitive deficits are well-researched in children, it is important to consider how these cognitive deficits are associated with reading and spelling abilities as children with dyslexia mature into adolescence and the extent to which cognitive deficits persist. The questions are then raised about what kinds of cognitive deficits exist in adolescents with dyslexia and which cognitive deficits are the most prominent features in Chinese adolescents with dyslexia. The present study, therefore, was among the first attempts to examine whether and to what extent adolescents with dyslexia could be differentially affected by various cognitive deficits associated with reading and spelling difficulties and their cognitive profiles.

To summarize, the present study investigated the extent to which four reading related cognitive skills would distinguish adolescent readers with dyslexia and their peers with same chronological age (CA) and matched reading level (RL). This study was designed with two major aims in mind. The first was to investigate the cognitive characteristics of Chinese adolescents with dyslexia in the four domains namely, 
visual-orthographic knowledge, morphological awareness, rapid naming, and verbal working memory. Its focus was on the characteristics of dyslexia in adolescence, and on how these four reading-related cognitive skills were associated with word reading, word spelling, and reading comprehension. With increasing literacy experience, do readers still have particular difficulties in these four domains as they mature into adolescence, and what kinds of profiles exist? Our second aim was to examine whether Chinese readers identified as dyslexic in their childhood would remain dyslexic in their adolescence. We expected that adolescents with dyslexia would experience cognitive deficits persisting from their childhood. According to the multiple deficits hypothesis, the number of cognitive deficits manifested by an individual was associated with the severity of his or her literacy difficulties (Ho et al., 2002). Thus, we anticipated that adolescents with difficulties across a wider range of cognitive tasks might also show greater reading and spelling difficulties. We also examined how four these cognitive skills were associated with word reading, word spelling, and reading comprehension performance among Hong Kong Chinese adolescents. Particularly, we were interested in the importance of rapid naming relative to other metalinguistic skills in relation to all three literacy measures because rapid naming was found to be a powerful predictor of Chinese literacy acquisition in children (e.g., Leong et al., 2008; McBride-Chang et al., 2008). On this basis, we expected that rapid naming tended to be strongly related to reading comprehension, word reading, and spelling in Chinese adolescents.

\section{Method}

\section{Participants}

The participants were 90 Chinese secondary school students in Hong Kong. All of these students had Chinese as their native language and had attended local primary schools where Cantonese was the teaching medium. Cantonese is a tonal dialect without formal written representation in that there is only a loose relationship between the spoken and written languages. There is no equivalent phonic system like pinyin for Cantonese, and no phonic instructions for Chinese are given in Hong Kong. Typically, Cantonese reading instruction proceeds by memorization of characters or the "look and say" method (Holm \& Dodd, 1996). Thirty participants ( 23 boys and 7 girls), all with normal intelligence (i.e., with IQ 85 or above), who had been identified in primary school years as having dyslexia by professional psychologists in accordance with the diagnostic criteria based on the Ho, Chan, Tsang, and Lee 's (2000) Hong Kong Test of Specific Learning Difficulties in Reading (HKT-SpLD) and Gardner's (1996) Test of Visual-Perceptual Skills (Non-motor) Revised (TVPS-R) were referred by the education authority and nongovernmental organizations. The HKT-SpLD battery, which was used to assess Cantonese-speaking Chinese children with dyslexia in the age range of 6 to 10 $1 / 2$ years, consisted of 12 tests including 3 literacy tests, 1 rapid naming test, 2 phonological awareness tests, 3 phonological memory tests, and 3 visual-orthographic knowledge tests. Also, the TVPS-R was used to test the children's visual 
Table 1 Characteristics of the three group of participants

\begin{tabular}{llllll}
\hline Characteristics & $\begin{array}{l}\text { Dyslexics } \\
(n=30)\end{array}$ & $\begin{array}{l}\text { CA controls } \\
(n=30)\end{array}$ & $\begin{array}{l}\text { RL controls } \\
(n=30) \\
M(\mathrm{SD})\end{array}$ & $F(2,87)$ & $\begin{array}{l}\text { Post hoc } \\
\text { comparison }\end{array}$ \\
\hline Age & $13.64(0.71)$ & $13.66(0.74)$ & $12.06(2.17)$ & $13.12^{* * * *}$ & $D>$ RL, $D=\mathrm{CA}$ \\
IQ & $10.57(2.31)$ & $10.97(1.52)$ & $10.53(2.19)$ & 0.42 & $D=\mathrm{RL}, D=\mathrm{CA}$ \\
Range of IQ & $7-17$ & $8-14$ & $7-16$ & & \\
\hline
\end{tabular}

$C A$ matched on chronological age, $R L$ matched on reading level, $D$ dyslexics

IQ was measured by a standardized oral vocabulary subtest from Hong Kong Wechsler Intelligence Scale for Children (HK-WISC)

perceptual and visual memory skills. To classify as dyslexics, their literacy composite score and at least one cognitive composite score had to be at least one standard deviation below the means of their respective age in the HKT-SpLD and TVPS-R.

Another 60 normally achieving students were recruited from one representative secondary school and primary school, respectively. These schools, which were located in a middle class neighborhood, were considered "average" and fairly representative of public schools in Hong Kong. None of these students had any history of learning difficulty or psychopathology in childhood. Thirty participants (13 boys and 17 girls) were selected from a secondary school for CA controls (matched on chronological age). These students were matched to the dyslexic students by the score of a standardized vocabulary test from Hong Kong Wechsler Intelligence Scale for Children (HK-WISC) and age and served as CA control group. This subtest could be used as a proxy for general ability or IQ as was demonstrated previously in related research (McBride-Chang \& Ho, 2005; McBride-Chang \& Kail, 2002).

Thirty readers ( 15 boys and 15 girls) were also selected from a primary school to match the participants with dyslexia on their Chinese word reading and a standardized vocabulary test from HK-WISC. This matching procedure used was similar to Ransby and Swanson's study (2003). They served as RL control group which was 1 year and 6 months younger than the dyslexic group. These 60 students were carefully selected to match the dyslexic group on age, reading level and IQ (see Table 1).

\section{Procedure}

Participants were administered 12 tasks including a standardized vocabulary test from HK-WISC, three literacy tests, two morphological awareness tests, two visualorthographic knowledge tests, two rapid naming tests, and two verbal working memory test. Except for the tasks of reading comprehension, dictation, and character matching, all other tasks were administered individually. The parents' or guardians' consents for students' participation were obtained before testing. Trained experimenters carried out all testing. 
General ability test

\section{Hong Kong Wechsler Intelligence Scale for Children (HK-WISC)}

The oral vocabulary subtest from HK-WISC was administered to assess general ability. The oral vocabulary subtest included 32 items. Participants were given the target words and required to explain the concepts of these words orally. The test was terminated if the participant scored 0 on five questions in a row. The vocabulary test was scored based on a standard scoring procedure from the local standardized norms established by Hong Kong Education Department in 1981. This task correlated .71 with the domain of verbal intelligence in the HK-WISC.

\section{Literacy tasks}

\section{Word reading}

Since no standardized Chinese word reading test was available for junior secondary students in Hong Kong, this task was constructed. A sample of 166 Chinese two-character words were chosen from the three sets of most popular Chinese language textbooks used in schools and various reading materials recommended from the Education and Manpower Bureau for the junior secondary school levels. These words were arranged in ascending order of difficulty and participants were asked to read them aloud one by one. One point was given when both characters of the words were read correctly.

\section{Reading comprehension}

Three stories were selected and modified based on the reading materials and texts recommended from the Education and Manpower Bureau. These stories with connected prose (one narrative, one descriptive, and one expository) were constructed and each of these was followed by several multiple-choice questions. The stories and questions were designed in ascending order of difficulty. Participants were asked to answer six multiple choice questions for each story. The maximum score on the test was 18 .

\section{Word dictation}

For this task, participants were orally presented with 54 two character words and then required to write them down on a paper. One point was given for each character written correctly.

Rapid naming tasks

\section{Rapid digit naming}

In this task, participants were presented with a sheet of paper containing all five digits $(2,4,6,7,9)$ each across five rows in different orders. Participants were 
required to name the numbers on the list as rapidly and accurately as possible. Each student named the list twice and the mean score was based on the average time taken across the two trials.

\section{Rapid letter naming}

Similar to the Rapid Digit Naming task, English letters were used because these stimuli were familiar to the students who were taught letters at their kindergarten. Participants were asked to read a series of lowercase letters (a, c, f, i, etc.) as quickly as they could. The students named the list twice and the average of these stimuli two times was calculated.

Verbal working memory test

\section{Backward digit span}

This task, which included a total of 14 sequences in lists starting from two digits to a maximum of eight digits was to assess the participants' short term verbal working memory. There were two sequences at each string length and the list length was increased by one. Participants listened to a string of digits and were asked to recall them in reverse sequence. The task was terminated after two consecutive failures at the same string length. Scoring was based on the total that was correctly reproduced.

\section{Non-word repetition}

This measure was used to test phonological working memory. There were 20 trials with three to eight Chinese syllables each in the memory test. Individual syllables were phonetically legal syllables in Cantonese but were nonsense characters (e.g. /bei5/, /tan5/, and /daai5/ were monosyllabic nonwords in Cantonese). The stimuli were presented through a CD player and the participants were asked to repeat orally the syllables in the presented order. One point was given for each correctly reproduced syllable in a trial.

\section{Morphological awareness tests}

\section{Morpheme discrimination}

This task was to measure students' understanding of the morpheme having different meanings in two morphemic words. This task included 19 items each consisting of 4 two-character words presented visually and orally. In each of the set, there was a character that shared the same sound and written form but with a different meaning when combined with the other characters. For example, a character 長, /tsoeng4/ is the common character in the words 長處 /tsoeng4 tsy2/, strength, 長篇, /tsoeng4 pin1/, long paragraph, 長路, /tsoeng4 lou6/, long road, and 長度, /tsoeng4 dou6/, length. For each set, participants were then asked to find the "odd" word. 
The correct response is 長處, /tsoeng4 tsy2/, strength because the character /tsoeng4/ in the word /tsoeng4 tsy2/ represents a different morpheme positive from the character /tsoeng4/ in the other three words long. One point was awarded for each correct response.

\section{Morpheme production}

This task was to examine the participants' ability to apply and integrate their morphological and contextual information in given contexts. In this task, 18 sentences with missing words were presented orally. The participants were told that they would hear some sentences with a word missing and they would have to replace the "blank". An example of a sentence would be “我地要對自己有信 ", We ought to have in ourselves and one of the possible correct response for this was 信心, confidence as 信, believe, trust was combined to the word 心, heart. A correct response was scored if the students gave the word response that fulfilled the semantic constraints of the position and made sense in the context of the sentence. The maximum score for the task was 36 .

Visual-orthographic knowledge tests

\section{Delayed copying}

This task, derived from the Delayed Copying Character task used by Pak, Cheng-Lai, Tso, Shu, Li, and Anderson (2005) was used to assess students' ability to retain character information and differentiate between the orthographic units in characters. Students were briefly shown 17 Chinese characters in which 14 pseudocharacters and 3 real characters as filler items were presented visually. Of the 14 pseudocharacters, half had 2 component characters (e.g., 発) and another half had 3 component characters (e.g., 戬). Each character was presented at a rate of 1 character per second and then was removed. Participants are required to count the numbers starting from 20 to 11 aloud before they reproduced the characters on paper with pencil. Asking the participants to count was to prevent them rehearsing the characters. One point was given for each correct component, and the total score for the task was 35 .

\section{Character matching}

This task was aimed at assessing the learners' knowledge about Chinese character structures. Students were asked to look at the target character and to find an identical one from the pool of 9 stimuli consisting of similar orthographic units and visual forms to the target characters. The stimuli were made up with the combination of five types of errors including illegal positions, inverted components, one component combining with different components, incorrect number of strokes, and incorrect orientation. For example, the target character 验 was presented simultaneously with the stimuli like 獫 (one component combining with a different component), 驗 (one component with missing strokes), 金 (the components in incorrect positions), 籡 (one component in an 
mirror orientation), and 劍 (the components in left/right reversed position). The test had a total of 18 items and one point was given for each correct item.

\section{Results}

Comparisons of the three groups for literacy and cognitive skills

The means, standard deviations, and reliability estimates for the tasks are given in Table 2. Overall, reliability of the tasks was acceptable (all .60 and above). A multivariate analysis of variance (MANOVA) was conducted to examine group differences on various literacy measures. A significant MANOVA difference was found for the effect of group differences, $F(6,170)=13.21, p<.001, \eta^{2}=.32$. Separate analyses of variance were conducted on each task (see Table 2). Using post hoc comparisons with Tukey's test revealed that the dyslexic group was significantly worse than the CA control group but similar to the RL control group. Both dyslexic group and RL group were matched with word reading. The only areas where the dyslexic group performed significantly worse than the RL group were reading comprehension and word spelling (or word dictation), indicating that they had experienced difficulties in these two domains. For the cognitive tasks, a MANOVA was also conducted to examine whether the effect of group was significant, $F$ (16, $160)=2.85, p<0.001, \eta^{2}=.30$ and Tukey's test was used to examine each task. The dyslexic group performed worse on most of the tasks than the CA group (see Table 2). They tended to perform less well than the RL group in the backward digit span task.

To further differentiate between dyslexic students and their peers for literacy and related cognitive tests, the effect size analysis that was used by Nicolson \& Fawcett (1995) is presented in Table 3. Effect size could be considered a standardized measure of group difference. The effect size in the standard scores was computed by subtracting the mean of the dyslexic group from that of both RL and CA group and dividing scores by the pooled standard deviation (Cohen, 1988). Cohen's tentative interpretation was used to explain the effect sizes $(d=0.2$ small, $d=0.5$ moderate, and $d=0.8$ large). Medium to large effect sizes were likely to be associated with more sensitive measures to discriminate well between group differences. As shown in Table 3, a comparably large effect size for word reading, reading comprehension, dictation, rapid letter and digit naming, backward digit span, morpheme production, delayed copying, and visual matching was found between the dyslexic students and CA controls whereas a medium effect size was obtained for the non-word repetition and morpheme discrimination test. Generally, the pattern of differences between the dyslexic and control groups was characterized by the dyslexic group's worse performance on measures of literacy and cognitive skills.

Correlations between literacy and cognitive skills

Two correlation analyses were conducted. Table 4 shows intercorrelations among all measures after partialling out the effects of age and IQ for the entire sample. All correlations were significant at the $.05, .01$, or .001 levels. The word reading, 
Table 2 Reliability coefficients, mean scores, and standard deviations on various literacy and cognitive tasks for the dyslexic group, CA control group, RL control group, and F values for group differences on various measures

\begin{tabular}{|c|c|c|c|c|c|c|c|c|c|}
\hline \multirow[t]{2}{*}{ Domain } & \multirow[t]{2}{*}{ Reliability } & \multicolumn{2}{|c|}{$\begin{array}{l}\text { Dyslexics } \\
(n=30)\end{array}$} & \multicolumn{2}{|c|}{$\begin{array}{l}\text { CA controls } \\
(n=30)\end{array}$} & \multicolumn{2}{|c|}{$\begin{array}{l}\text { RL controls } \\
(n=30)\end{array}$} & \multirow[t]{2}{*}{$F(2,87)$} & \multirow[t]{2}{*}{$\begin{array}{l}\text { Post hoc } \\
\text { comparison }\end{array}$} \\
\hline & & $M$ & SD & $M$ & SD & $M$ & SD & & \\
\hline \multicolumn{10}{|l|}{ Literacy skills } \\
\hline Word reading & .98 & 74.77 & 30.62 & 105.70 & 16.05 & 76.23 & 31.49 & $12.53 * * *$ & $\begin{array}{l}D=\mathrm{RL} \\
D<\mathrm{CA}\end{array}$ \\
\hline Word dictation & .96 & 23.67 & 14.57 & 56.30 & 17.31 & 38.60 & 23.44 & $22.62 * * *$ & $\begin{aligned} D & <\mathrm{RL} \\
D & <\mathrm{CA}\end{aligned}$ \\
\hline $\begin{array}{l}\text { Reading } \\
\text { comprehension }\end{array}$ & .64 & 8.43 & 3.55 & 13.57 & 1.98 & 11.30 & 3.57 & $20.34 * * *$ & $\begin{array}{r}D<\mathrm{RL} \\
D<\mathrm{CA}\end{array}$ \\
\hline \multicolumn{10}{|l|}{ Cognitive skills } \\
\hline \multicolumn{10}{|c|}{ Visual-orthographic knowledge tasks } \\
\hline Delayed copying & .72 & 11.50 & 4.84 & 15.47 & 4.80 & 12.33 & 5.33 & $5.23 * *$ & $\begin{aligned} D=\mathrm{RL} \\
D<\mathrm{CA}\end{aligned}$ \\
\hline $\begin{array}{l}\text { Character } \\
\text { matching }\end{array}$ & .67 & 13.73 & 2.53 & 15.87 & 1.41 & 14.83 & 3.07 & $5.74 * *$ & $\begin{array}{l}D=\mathrm{RL} \\
D<\mathrm{CA}\end{array}$ \\
\hline \multicolumn{10}{|l|}{ Rapid naming tasks } \\
\hline $\begin{array}{l}\text { Rapid digit } \\
\text { naming }\end{array}$ & .90 & 37.11 & 12.60 & 26.52 & 4.85 & 31.90 & 8.77 & $9.73 * * *$ & $\begin{array}{l}D=\mathrm{RL}, \\
D>\mathrm{CA}\end{array}$ \\
\hline $\begin{array}{l}\text { Rapid letter } \\
\text { naming }\end{array}$ & .94 & 56.90 & 18.00 & 41.10 & 8.20 & 49.63 & 15.08 & $9.10 * * *$ & $\begin{array}{l}D=\mathrm{RL}, \\
D>\mathrm{CA}\end{array}$ \\
\hline \multicolumn{10}{|c|}{ Morphological awareness tasks } \\
\hline $\begin{array}{l}\text { Morpheme } \\
\text { discrimination }\end{array}$ & .60 & 10.83 & 3.44 & 12.80 & 2.63 & 10.47 & 2.92 & $5.19 * *$ & $\begin{array}{l}D=\mathrm{RL} \\
D<\mathrm{CA}\end{array}$ \\
\hline $\begin{array}{l}\text { Morpheme } \\
\text { production }\end{array}$ & .80 & 28.13 & 4.49 & 31.30 & 2.73 & 25.87 & 7.04 & $8.68 * * *$ & $\begin{array}{l}D=\mathrm{RL} \\
D<\mathrm{CA}\end{array}$ \\
\hline \multicolumn{10}{|c|}{ Verbal working memory task } \\
\hline $\begin{array}{l}\text { Backward digit } \\
\text { span }\end{array}$ & .74 & 5.20 & 1.94 & 7.70 & 2.39 & 6.67 & 2.78 & $8.24 * * *$ & $\begin{aligned} D & <\mathrm{RL} \\
D & <\mathrm{CA}\end{aligned}$ \\
\hline $\begin{array}{l}\text { Non-word } \\
\text { repetition }\end{array}$ & .72 & 7.2 & 2.3 & 8.91 & 2.92 & 7.51 & 2.85 & $7.23 * *$ & $\begin{array}{c}D=\mathrm{RL} \\
D<\mathrm{CA}\end{array}$ \\
\hline
\end{tabular}

$C A$ matched on chronological age, $R L$ matched on reading level, $D$ dyslexics

$* p<.05 ; * * p<.01 ; * * * p<.001$

reading comprehension, and word dictation was significantly and moderately associated with all cognitive measures.

In Table 5, the partial correlations were also conducted to examine the severity of reading and spelling difficulties within the dyslexic group. The reading and spelling measures were highly correlated even after the effects of age and IQ were controlled (all $r \mathrm{~s}>.38$, all $p \mathrm{~s}<.05$ ). The word reading, reading comprehension, and word dictation were correlated with all of the cognitive tasks. The result suggests that if the students have severe difficulties in reading, they appear to have similar great difficulties in spelling. 
Table 3 Mean effect sizes (Cohen's $d$ ) for dyslexic adolescents versus CA controls and RL controls

\begin{tabular}{lll}
\hline Variable & \multicolumn{2}{l}{ Dyslexic adolescents vs. } \\
\cline { 2 - 3 } & CA controls ${ }^{\mathrm{a}}$ & \\
\hline Literacy skills & & RL controls $^{\mathrm{b}}$ \\
$\quad$ Word reading & -1.27 & -0.05 \\
$\quad$ Word dictation & -2.04 & -0.77 \\
Reading comprehension & -1.79 & -0.81 \\
Cognitive skills & & \\
Visual-orthographic knowledge tasks & & \\
Delayed copying & -0.82 & -0.16 \\
Character matching & -1.04 & -0.39 \\
Rapid naming tasks & & \\
Rapid digit naming & 1.11 & 0.48 \\
Rapid letter naming & 1.13 & 0.44 \\
Morphological awareness tasks & & 0.11 \\
Morpheme discrimination & -0.64 & 0.38 \\
$\quad$ Morpheme production & -0.85 & \\
Verbal working memory task & & -0.61 \\
Backward digit span & -1.15 & -0.12 \\
Non-word repetition & -0.65 & \\
\hline
\end{tabular}

${ }^{\mathrm{a}}\left(\right.$ Mean $_{\text {Dyslexic }}-$ Mean $\left._{\mathrm{CA} \text { control }}\right) /$ pooled standard deviation

${ }^{\mathrm{b}}\left(\right.$ Mean $_{\text {Dyslexic }}-$ Mean $\left._{\text {RL control }}\right) /$ pooled standard deviation

Predicting literacy ability from cognitive skills

Hierarchical multiple regressions were performed on the combined data from all of the three age groups to examine the extent to which the verbal working memory, rapid naming, visual-orthographic knowledge, and morphological awareness measures explained variability in word reading, reading comprehension, and word spelling. These metalingusitic measures were entered with verbal working memory first because it is best established as a strong correlate of reading across orthographies (e.g., Leong et al., 2008; Leong, Hau, Tse, \& Loh, 2007), visual-orthographic skills and morphological awareness were second and third, because they have been shown to be strongly related to literacy ability (e.g., McBride-Chang, Shu, Zhou, Wat \& Wagner, 2003; Shu et al., 2006), and finally rapid naming. Rapid naming was entered as the final cognitive correlate because it is a powerful correlate of reading ability and a strong predictor of both concurrent and future reading development in Chinese (e.g., Georgiou, Parrila, \& Kirby, 2006; Ho \& Lai, 1999). Bowers and Newby-Clark (2002) have also suggested that rapid naming plays a crucial role in lexical or whole-word processing. Therefore, the control variables of age and IQ were entered as the first block into each regression analysis. The measures of verbal working memory, visualorthographic knowledge, morphological awareness, and rapid naming were then entered in the second, third, fourth, and fifth blocks, respectively. 


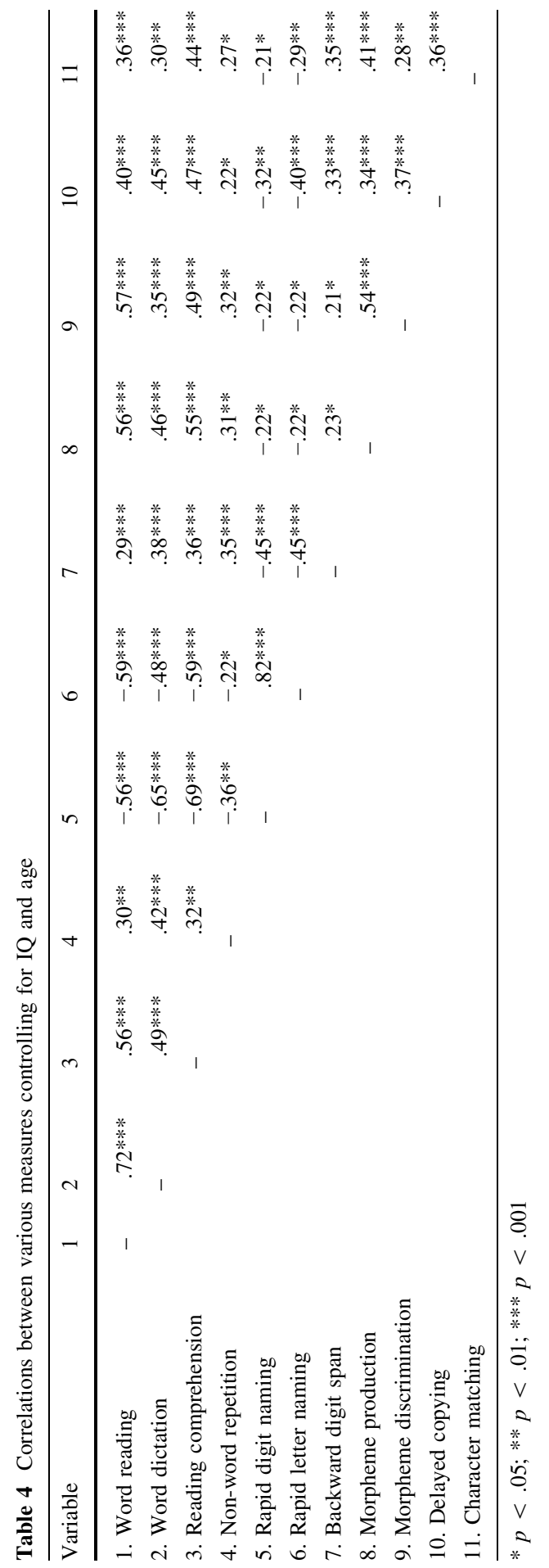


Table 5 A matrix of partial correlation coefficients for the dyslexic adolescents $(n=30)$ after controlling for the differences in age and IQ

\begin{tabular}{lccc}
\hline & Word reading & Reading comprehension & Word dictation \\
\hline Word reading & & $0.39^{*}$ & $0.65^{* * *}$ \\
Word dictation & $0.65^{* * *}$ & $0.38^{*}$ & \\
Reading comprehension & $0.43^{*}$ & & $0.41^{*}$ \\
Non-word repetition & $0.39^{*}$ & $0.42^{*}$ & $0.43^{*}$ \\
Rapid digit naming & $-0.64^{* * *}$ & $-0.57^{* *}$ & $-0.69^{* * *}$ \\
Rapid letter naming & $-0.46^{* *}$ & $-0.54^{* *}$ & $-0.46^{* *}$ \\
Backward digit span & $0.44^{*}$ & $0.38^{*}$ & $0.42^{*}$ \\
Morpheme production & $0.48^{*}$ & $0.47^{*}$ & $0.39^{*}$ \\
Morpheme discrimination & $0.69^{* * *}$ & $0.56^{* * *}$ & $0.41^{*}$ \\
Delayed copying & $0.39^{*}$ & $0.49^{* *}$ & $0.53^{* *}$ \\
Character matching & $0.40^{*}$ & $0.39^{*}$ & $0.43^{*}$ \\
\hline
\end{tabular}

$* p<.05 ; * * p<.01 ; * * * p<.001$

Table 6 Hierarchical regression analyses predicting word reading, word dictation, and reading comprehension using the four cognitive skills

\begin{tabular}{|c|c|c|c|c|}
\hline Variable/step & Final beta & Total $R^{2}$ & $R^{2}$ change & $F$ change \\
\hline \multicolumn{5}{|l|}{ Word reading } \\
\hline Age/IQ & $0.52 * * * / 0.36 * * *$ & 0.36 & 0.36 & $24.15 * * *$ \\
\hline Non-word repetition/backward digit span & $0.03 / 0.21^{*}$ & 0.40 & 0.05 & $3.33^{*}$ \\
\hline Delayed copying/character matching & $0.27 * * * / 0.11$ & 0.48 & 0.07 & $5.83 * *$ \\
\hline Morpheme production/morpheme discrimination & $0.41 * * * / 0.30 * * *$ & 0.65 & 0.17 & $19.64 * * *$ \\
\hline Rapid digit naming/rapid letter naming & $-0.30 /-0.26$ & 0.70 & 0.05 & $6.74 * *$ \\
\hline \multicolumn{5}{|l|}{ Word spelling (or dictation) } \\
\hline Age/IQ & $0.39 * * * / 0.19 *$ & 0.17 & 0.17 & $9.10^{* * * *}$ \\
\hline Non-word repetition/backward digit span & $-0.10 /-0.31 * * *$ & 0.26 & 0.08 & $4.79^{*}$ \\
\hline Delayed copying/character matching & $0.35 * * * / 0.14$ & 0.38 & 0.12 & $8.33 * *$ \\
\hline Morpheme production/morpheme discrimination & $0.43 * * * / 0.06$ & 0.46 & 0.08 & $5.76^{* *}$ \\
\hline Rapid digit naming/rapid letter naming & $-0.09 /-0.23$ & 0.52 & 0.06 & $5.13 * *$ \\
\hline \multicolumn{5}{|l|}{ Reading comprehension } \\
\hline Age/IQ & $0.20 / 0.34 * * *$ & 0.14 & 0.14 & $6.79 * *$ \\
\hline Non-word repetition/backward digit span & $-0.03 / 0.29 * * *$ & 0.21 & 0.07 & $3.96^{*}$ \\
\hline Delayed copying/character matching & $0.33 * * * / 0.29 * * *$ & 0.40 & 0.19 & $13.51 * * *$ \\
\hline Morpheme production/morpheme discrimination & $0.41 * * * / 0.21 *$ & 0.53 & 0.12 & $10.58^{* * *}$ \\
\hline Rapid digit naming/rapid letter naming & $-0.19 /-0.23$ & 0.62 & 0.09 & $10.33^{* *}$ \\
\hline
\end{tabular}

$* p<.05 ; * * p<.01 ; * * * p<.001$

As shown in Table 6, the regression analysis indicated that the four cognitive skills explained significant variables in word reading ability: age and IQ $\left(R^{2}=0.36\right)$, backward digit span and non-word repetition (additional variance explained $=0.40$ ), character matching and delayed copying (additional variance explained $=0.48$ ), 
morpheme discrimination and morpheme production (additional variance explained $=0.65$ ), and rapid digit naming and rapid letter naming (additional variance explained $=0.70$ ). For predicting word spelling or dictation, the regression analysis showed age and IQ $\left(R^{2}=0.17\right)$, backward digit span and non-word repetition (additional variance explained $=0.26$ ), character matching and delayed copying (additional variance explained $=0.38$ ), morpheme discrimination and morpheme production (additional variance explained $=0.46$ ), and rapid digit naming and rapid letter naming (additional variance explained $=0.52$ ). Table 5 also shows that the four cognitive skills contributed significant variables to reading comprehension: age and IQ $\left(R^{2}=0.14\right)$, backward digit span and non-word repetition (additional variance explained $=0.21$ ), character matching and delayed copying (additional variance explained $=0.40$ ), morpheme discrimination and morpheme production (additional variance explained $=0.53$ ), and rapid digit naming and rapid letter naming (additional variance explained $=0.62$ ). In the present findings, the rapid naming explained a significant amount of variance in word reading, word spelling, and reading comprehension beyond other variables. Overall, the results showed that the four cognitive skills were strongly associated with word reading, word spelling, and reading comprehension.

\section{Discussion}

The present investigation was among the first attempts to establish literacy and cognitive measures that might distinguish Chinese Cantonese-speaking adolescents with and without dyslexia. We compared adolescent readers diagnosed with childhood dyslexia and normal achieving readers and found that the dyslexic group performed less well than the control groups on a range of literacy and cognitive tasks. The dyslexic adolescents were significantly weaker than controls in all measures of literacy as well as in the four cognitive measures: rapid naming, visual-orthographic knowledge, morphological awareness, and verbal working memory. Our findings extended the existing work on multiple cognitive deficits (e.g., Bruck, 1990, 1992; Miller-Shaul, 2005; Shaywitz et al., 1999; Wolf \& Katzir-Cohen, 2001) that might underlie reading and spelling mechanisms in Chinese speaking communities. Furthermore, our results showed that verbal working memory, rapid naming, visualorthographic knowledge, and morphological awareness were significantly associated with word reading, word spelling, and reading comprehension in regression analyses.

The present findings indicated that dyslexic students tended to be poorer in both reading and spelling when compared with their peers. Also, dyslexic students appeared to show more difficulties in reading comprehension and spelling. Our results found significant differences between the dyslexic and control group on the reading and spelling tasks, with the effect sizes for reading comprehension, word reading, and spelling being reasonably large, above 0.8 (Cohen, 1988). These results were comparable with the previous research showing significant impairments of reading comprehension and spelling in dyslexic adolescents (Everatt, 1997; Simmons \& Singleton 2000). This suggests that the adolescent readers with dyslexia have difficulty decoding the written word and this affects comprehension. Their difficulties 
in spelling could also be affected by this deficit. The results of this study cast doubt on the suggestion stating the dyslexic adolescents can compensate for their reading and spelling problems to varying degrees after years of print exposure and remedial teaching (Bruck, 1992; Snowling, 2000). Consistent with current research (Lehtola \& Lehto, 2000; Shaywitz et al., 1999), the present findings showed the persistence of reading and spelling difficulties in adolescence.

There were some differences between the dyslexic and control groups in certain cognitive skills. Many dyslexic adolescents were found to be slower in rapid naming tasks than the control group. It was also found that rapid naming was strongly associated with reading and spelling in dyslexic students. This may reflect difficulties with generally weak phonological representations, less automatic processes of extraction and induction of orthographic patterns, less efficient lexical access, and thus be considered one underlying cause of poor reading, particularly in Chinese. Rapid naming has been viewed as an important part of literacy acquisition and failure since Chinese script has relatively arbitrary associations between print and sound (e.g., Ho \& Lai, 1999; McBride-Chang \& Ho, 2000). Such difficulties of speed processing are likely to reflect slowness in developing stable and strong orthographic and multi-morphemic representations. Consistent with the current findings, Chung, Ho, Chan, Tsang, \& Lee (2010) also found that rapid naming deficit was the most dominant type of cognitive deficit and major type of difficulties in the Chinese adolescents with dyslexia. About 35.2\% of dyslexic adolescents in their study demonstrated deficits in rapid naming. Furthermore, it was found that more severe Chinese dyslexics are particularly more impaired in rapid naming than are less severe dyslexics. Additionally, these results were compatible with the findings of Chinese children with dyslexia (Ho et al., 2002, 2004) reporting that over half of the dyslexic children had rapid naming deficit, making it one of the most prominent problems faced by Chinese readers with dyslexia. Taken together, the present and previous findings have shown that rapid naming deficit is more common in Chinese dyslexia and seems to be a major contributor to literacy failures in Chinese. Rapid naming deficit may remain problematic for dyslexic adolescents and characterize readers with dyslexia in adolescence. As Korhonen (1995) suggested, deficient rapid naming persists over a number of years and it is likely that difficulty in rapid naming is an ongoing problem for many dyslexic readers (Felton et al., 1990).

Differences in visual-orthographic knowledge were found between the dyslexic and control groups and this orthographic processing was strongly associated with reading and spelling abilities in dyslexic students. Visual-orthographic knowledge appears to be a major difficulty for these readers. It seems that for dyslexic adolescents, knowledge of orthographic structure, and implicit knowledge of radical positions has not yet been mastered. Perhaps this is due to the nature of Chinese orthography and the way Hong Kong Chinese students learn to read. In Hong Kong, Chinese characters are generally taught by the whole word approach without much emphasis on the characters' orthographic structure. Also, it is noteworthy that traditional Chinese character script used in Hong Kong may further contribute to the reading and spelling problem. Traditional characters are formed with a greater number of strokes and complex forms than the simplified characters used in China. In line with the present research (Ho et al., 2002, 2004), the dyslexic students' 
literacy problems are coupled with deficits in visual-orthographic knowledge. The present data are in line with the research demonstrating that the visual-orthographic processing ability of dyslexics does not improve over time (Bruck, 1998; Corcos \& Willows, 1993).

The dyslexic and control groups were found to differ substantially in morphological awareness and verbal working memory that were moderately linked with reading and spelling abilities in dyslexic adolescents. The dyslexic readers tended to have difficulties in morphological awareness because many homophones and words are formed with multi-morphemes in Chinese script. They are likely to have difficulty in identifying and discriminating morphemes, manipulating the morphemic structure and generalizing morpheme meaning. They may be unaware of the role of radicals and morphological relations, storing words in isolation rather than network forms. Given these possible reasons, poor morphological awareness may prevent them from forming clear and stable semantic representations of morphemes and multimorphemic networks which could lead to a potential problem in developing reading and spelling over time. The dyslexic adolescents also had difficulty in verbal working memory that could be attributed to a deficiency in a short-term store for phonological information. This suggests that poor verbal working memory may impair functioning of the phonological store which is likely to hinder the development of stable associations between visual symbols and units of speech sounds and learning of verbal vocabulary. Thus the acquisition of literacy skills could be hampered. Consistent with the previous studies (Gathercole, Pickering, Knight, \& Stegmann, 2004; Zhang et al., 1998), Chinese dyslexic adolescents seem to have difficulties in keeping sounds in their verbal working memory and, in turn, these problems may reflect some underlying weakness in the development of graphic-sound associations, multimorpheme words, and stable orthographic knowledge, adversely affecting literacy acquisition. The association of morphological awareness, and verbal working memory deficits to reading and spelling abilities in the present study has also been demonstrated in some previous studies (e.g., Shu et al., 2006; Zhang et al., 1998).

In the present study, verbal working memory, visual-orthographic knowledge, morphological awareness, and rapid naming, were more strongly related to word reading, reading comprehension, and word spelling when all cognitive skills were included in regression equations, supplying additional evidence of the potential importance of these metalinguistic tasks for literacy skills in Chinese. Particularly, rapid naming emerged as important and powerful predictor for explaining variance in overall reading and spelling performance among Chinese adolescents. The findings on rapid naming which showed essential for understanding Chinese literacy acquisition in adolescents extended previous research on the contribution of rapid naming to literacy achievement in Chinese children (Ho \& Lai, 1999; Leong et al., 2008; Shu et al., 2006). It can be seen in the present study that verbal working memory, rapid naming, morphological awareness, and visual-orthographic knowledge are the potential important predictors for Chinese literacy acquisition, suggesting that these four cognitive skills could saliently affect both reading and spelling abilities in Chinese adolescents. Our results are also consistent with previous research demonstrating the cognitive skills in verbal working memory, rapid naming, orthographic knowledge, and morphological awareness explained variability in 
reading and spelling performance in Chinese readers (Chan et al., 2006; Leong et al., 2008; McBride-Chang et al., 2008; Shu et al., 2006).

This study certainly had many limitations that need to be considered. First, across many measures administered, the dyslexic students did not differ in performance levels from their reading level controls. Similar findings have been reported in past research (e.g., Chung et al., 2010; Ho et al., 2002; Ransby \& Swanson, 2003). However, this finding is somewhat problematic for arguing about causality in reading. It is likely that dyslexia implies a general lag, rather than a permanent deficit, in a variety of cognitive skills among Chinese students. Support for this conclusion can only be obtained through longitudinal studies, some of which follow dyslexics into adulthood. Second, our data were limited to examine the cognitive profile of students with dyslexia in junior secondary schools. We were unable to obtain these students' profile identified as having dyslexia in their primary school year. Thus, we do not know the extent to which the variability of individuals' profile has been changed over time. In longitudinal studies of dyslexia (e.g., Badian, 1999; Shaywitz et al., 1992), it was found that individuals' profile could exhibit a year-toyear variability due to the possible factors such as developmental change and increasing exposure of literacy experience. With a larger sample, we may be able to examine whether the students identified as having dyslexia in early grades will continue to have problems in later grades and what are their characteristics. Further work is required to investigate the stability of classification over time. Also, it is not known to what extent factors such as special coaching or remedial teaching play a role in discrepant findings because the present studies did not collect this information. Nevertheless, future studies should take these factors into account when it is practical to do so. Third, the present study's limited and specific set of cognitive skills was unlikely to offer broad understanding of literacy problems in Chinese. Other aspects of cognitive ability like semantic and syntactic skills might be incorporated in future research. Fourth, our present sample size was small so further work needs to be conducted with a larger sample to confirm the present findings. Fifth, the data was limited to readers from Hong Kong. Because of the difference between simplified and traditional character scripts and literacy instruction among the Chinese readers in Hong Kong, mainland China, and Taiwan, the extent to which our findings can be generalized across Chinese societies is not yet fully understood. Finally, a comparison of reading difficulties in the different orthographies might be worthwhile because in alphabetic languages, phonological deficit seems to be a major deficit as compared to non-alphabetic languages like Chinese. Future research is needed to investigate this matter.

Despite these limitations, the present study has offered new insights into Chinese Cantonese-speaking adolescents diagnosed with childhood dyslexia and indicates areas for future research. The present study is among the first to focus on the characteristics of dyslexia in adolescence and reveals that Chinese dyslexic adolescents demonstrate continuing problems in reading and spelling abilities. Our study has demonstrated at least four potentially important aspects of cognitive skills in rapid naming, verbal working memory, morphological awareness, and visualorthographic knowledge for Chinese literacy acquisition. These cognitive skills seem to contribute significantly to word reading, word spelling, and reading comprehension 
performance. The present findings support the generality of this statement that dyslexia is a chronic and persistent difficulty across all ages and scripts.

Acknowledgements This research was supported in part by Hong Kong Jockey Club of Charities Trust, Quality Education Fund (2003/0740), and General Research Fund from Research Grants Council (HKIED: 8402/05H, 840308) to Kevin. K. H. Chung. The authors thank Sue Lea, Prof. Joshi, the editor, and two anonymous reviewers for their suggestions to improve the manuscript. We would also like to thank the support of research assistants, teachers, and students of the participating schools.

Open Access This article is distributed under the terms of the Creative Commons Attribution Noncommercial License which permits any noncommercial use, distribution, and reproduction in any medium, provided the original author(s) and source are credited.

\section{References}

Baddeley, A., Gathercole, S., \& Papagno, C. (1998). The phonological loop as a language learning device. Psychological Review, 105, 158-173.

Badian, N. A. (1999). Reading disability defined as a discrepancy between listening and reading comprehension: A longitudinal study of stability, gender differences, and prevalence. Journal of Learning Disabilities, 32, 138-148.

Ben-Dror, I., Bentin, S., \& Frost, R. (1995). Semantic, phonologic, and morphologic skills in reading disabled and normal children: Evidence from perception and production of spoken Hebrew. Reading Research Quarterly, 30, 876-893.

Bowers, P. G., \& Newby-Clark, E. (2002). The role of naming speed within a model of reading acquisition. Reading and Writing: An interdisciplinary Journal, 15, 109-126.

Bruck, M. (1990). Word-recognition skills of adults with childhood diagnoses of dyslexia. Developmental Psychology, 26, 439-454.

Bruck, M. (1992). Persistence of dyslexics' phonological awareness deficits. Developmental Psychology, 28, 874-886.

Bruck, M. (1998). Outcomes of adults with childhood histories of dyslexia. Mahwah, NJ, USA: Lawrence Erlbaum Associates Publishers.

Carlisle, J. F. (1995). Morphological awareness and early reading achievement. In L. Feldman (Ed.), Morphological aspects of language processing (pp. 189-209). Hillsdale, NJ: Erlbaum.

Casalis, S., \& Louis-Alexandre, M.-F. (2000). Morphological analysis, phonological analysis and learning to read French: A longitudinal study. Reading and Writing: An interdisciplinary Journal, 12, 303335 .

Catts, H. W., \& Kamhi, A. G. (2005). Defining reading disabilities. In H. W. Catts \& A. G. Kamki (Eds.), Language and reading disabilities (pp. 50-67). Boston, MA: Allyn \& Bacon.

Chan, D. W., Ho, C. S. H., Tsang, S. M., Lee, S. H., \& Chung, K. K. H. (2006). Exploring the readingwriting connection in Chinese children with dyslexia in Hong Kong. Reading and Writing: An interdisciplinary Journal, 19, 543-561.

Chung, K. K. H., Ho, C. S. H., Chan, D. W., Tsang, S. M., \& Lee, S. H. (2010). Cognitive profiles of Chinese adolescents with dyslexia. Dyslexia, 16, 2-23.

Cohen, J. (1988). Statistical power analysis for the behavioral sciences (2nd ed.). Hillsdale, NJ: Lawrence Earlbaum Associates.

Corcos, E., \& Willows, D. (1993). The role of visual processing in good and poor readers' utilization of orthographic information in letter strings. In S. F. Wright \& R. Groner (Eds.), Facets of dyslexia and its remediation (pp. 95-106). Amsterdam, The Netherlands: Elsevier.

de Jong, P. F., \& van der Leij, A. (2003). Developmental changes in the manifestation of a phonological deficit in dyslexic children learning to read a regular orthography. Journal of Educational Psychology, 95, 22-40.

DeFrancis, J. (1989). Visible speech: The diverse oneness of writing systems. Honolulu, HI: University of Hawaii Press. 
Everatt, J. (1997). The abilities and disabilities associated with adult developmental dyslexia. Journal of Research in Reading, 20, 13-21.

Feldman, L. B., \& Siok, W. W. T. (1997). The role of component function in visual recognition of Chinese characters. Journal of Experimental Psychology. Learning, Memory, and Cognition, 23, 776-781.

Feldman, L. B., \& Siok, W. W. T. (1999). Semantic radicals in phonetic compounds: Implications for visual character recognition in Chinese. In A. J. Wang, W. Inhoff, \& H. C. Chen (Eds.), Reading Chinese script: A cognitive analysis (pp. 19-36). Mahwah, NJ: Lawrence Erlbaum Associates.

Felton, R. H., Naylor, C. E., \& Wood, B. E. (1990). Neuropsychological profile of adult dyslexic children. Brain and Language, 39, 485-497.

Fowler, C. A. (1991). Auditory perception is not special: We see the world, we feel the world, we hear the world. Journal of the Acoustical Society of America, 89, 2910-2915.

Gardner, M. F. (1996). Test of visual-perceptual skills (non-motor)-revised. Hydesville, CA: Psychological and Educational Publications.

Gathercole, S. E., Pickering, S. J., Knight, C., \& Stegmann, Z. (2004). Working memory skills and educational attainment: Evidence from National Curriculum assessments at 7 and 14 years of age. Applied Cognitive Psychology, 40, 1-16.

Georgiou, G. K., Parrila, R., \& Kirby, J. (2006). Rapid naming speed components and early reading acquisition. Scientific Studies of Reading, 10, 199-220.

Ho, C. S. H., Chan, D. W., Chung, K. K. H., Lee, S. H., \& Tsang, S. M. (2007). In search of subtypes of Chinese developmental dyslexia. Journal of Experimental Child Psychology, 97, 61-83.

Ho, C. S. H., Chan, D. W. O., Lee, S. H., Tsang, S. M., \& Luan, V. H. (2004). Cognitive profiling and preliminary subtyping in Chinese developmental dyslexia. Cognition, 91, 43-75.

Ho, C. S. H., Chan, D. W., Tsang, S., \& Lee, S. (2000a). The Hong Kong test of specific learning difficulties in reading and writing (HKT-SpLD) manual. Hong Kong: Hong Kong Specific Learning Difficulties Research Team.

Ho, C. S. H., Chan, D. W., Tsang, S., \& Lee, S. (2002). The cognitive profile and multiple-deficit hypothesis in Chinese developmental dyslexia. Developmental Psychology, 38, 543-553.

Ho, C. S. H., \& Lai, D. N.-C. (1999). Naming-speed deficits and phonological memory deficits in Chinese developmental dyslexia. Learning and Individual Differences, 11, 173-186.

Ho, C. S. H., Law, T. P. S., \& Ng, P. M. (2000b). The phonological deficit hypothesis in Chinese developmental dyslexia. Reading and Writing: An interdisciplinary Journal, 13, 57-79.

Holm, A., \& Dodd, B. (1996). The effect of first written language on the acquisition of English literacy. Cognition, 59, 119-147.

Hu, C. F., \& Catts, H. W. (1998). The role of phonological processing in early reading ability: What we can learn from Chinese. Scientific Studies of Reading, 2, 55-79.

Hulme, C. (1981). The effects of manual tracing on memory in normal and retarded readers: Some implications for multi-sensory teaching. Psychological Research, 43, 179-191.

Hultquist, A. M. (1997). Orthographic processing abilities of adolescents with dyslexia. Annals of Dyslexia, 47, 89-114.

Kang, J. S. (1993). Analysis of semantics of semantic-phonetics compound characters in modern Chinese. In Y. Chen (Ed.), Information analysis of usage of characters in modern Chinese. Shanghai: Shanghai Education Publisher.

Korhonen, T. T. (1995). The persistence of rapid naming problems in children with reading disabilities: A nine year follow-up. Journal of Learning Disabilities, 28, 232-239.

Lehtola, R., \& Lehto, J. E. (2000). Assessing dyslexia in Finnish high-school students: A pilot study. European Journal of Special Needs Education, 15, 255-263.

Leong, C. K. (1999). Phonological and morphological processing in adult students with learning/reading disabilities. Journal of Learning Disabilities, 32, 224-238.

Leong, C. K., Hau, K. T., Tse, S. K., \& Loh, K. Y. (2007). Component skills of text comprehension in less competent Chinese comprehenders. Annuals of Dyslexia, 57, 75-97.

Leong, C. K., Tse, S. K., Loh, K. Y., \& Hau, K. T. (2008). Text comprehension in Chinese children: Relative contribution of verbal working memory, pseudoword reading, rapid automatized naming, and onset-rime phonological segmentation. Journal of Educational Psychology, 100, 135-149.

Manis, F. R., Seidenberg, M. S., \& Doi, L. M. (1999). See Dick RAN: Rapid naming and the longitudinal prediction of reading subskills in first and second graders. Scientific Studies of Reading, 3, 129-157.

Mattingly, I. G. (1984). Reading, linguistic awareness, and language acquisition. In R. V. J. Downing (Ed.), Language awareness and learning to read (pp. 9-26). New York, NY: Springer. 
Maughan, B., \& Hagell, A. (1996). Poor readers in adulthood: Psych-social functioning. Development and Psychopathology, 8, 457-476.

McBride-Chang, C. (1995). What is phonological awareness? Journal of Educational Psychology, 87, 179-192.

McBride-Chang, C., \& Ho, C. S. H. (2000). Developmental issues in Chinese children's character acquisition. Journal of Educational Psychology, 92, 50-55.

McBride-Chang, C., \& Ho, C. S. H. (2005). Predictors of beginning reading in Chinese and English: A 2-Year Longitudinal Study of Chinese Kindergartners. Scientific studies of Reading, 9, 117-144.

McBride-Chang, C., \& Kail, R. V. (2002). Cross-cultural similarities in the predictors of reading acquisition. Child Development, 73, 1392-1407.

McBride-Chang, C., Shu, H., Zhou, A., Wat, C. P., \& Wagner, R. K. (2003). Morphological awareness uniquely predicts young children's Chinese character recognition. Journal of Educational Psychology, 95, 743-751.

McBride-Chang, C., Tong, X., Shu, H., Wong, A. M. Y., Leung, K., \& Tardif, T. (2008). Syllable, phoneme, and tone: Psycholinguistic units in early Chinese and English word recognition. Scientific Studies of Reading, 12, 171-194.

Miller-Shaul, S. (2005). The characteristics of young and adult dyslexics readers on reading and reading related cognitive tasks as compared to normal readers. Dyslexia, 11, 132-151.

Nation, K., \& Snowling, M. (1998). Individual differences in contextual facilitation: Evidence from dyslexia and poor reading comprehension. Child Development, 69, 996-1011.

Nicolson, R. I., \& Fawcett, A. J. (1995). Dyslexia is more than a phonological disability. Dyslexia, 1, 19-36.

Packard, J. L. (2000). The morphology of Chinese: A linguistic and cognitive approach. Cambridge, England: Cambridge University Press.

Pak, A. K. H., Cheng-Lai, A., Tso, I. F., Shu, H., Li, W., \& Anderson, R. C. (2005). Visual chunking skills of Hong Kong children. Reading and Writing: An interdisciplinary Journal, 18, 437-454.

Ransby, M. J., \& Swanson, H. L. (2003). Reading comprehension skills of young adults with childhood diagnoses of dyslexia. Journal of Learning Disabilities, 36, 538-555.

Shaywitz, S. E., Escobar, M. D., Shaywitz, B. A., Fletcher, J. M., \& Makuch, R. (1992). Evidence that dyslexia may represent the lower tail of a normal distribution of reading ability. New England Journal of Medicine, 326, 145-150.

Shaywitz, S. E., Fletcher, J., Holahan, J., Shneider, A., Marchione, K., Stuebing, K., et al. (1999). Persistence of dyslexia: The Connecticut Longitudinal Study at adolescence. Pediatrics, 104, 13511359.

Shaywitz, S. E., \& Shaywitz, B. A. (2005). Dyslexia (specific reading disability). Biological Psychiatry, 57, 1301-1309.

Shu, H., \& Anderson, R. C. (1997). Role of radical awareness in the character and word acquisition of Chinese children. Reading Research Quarterly, 32, 78-89.

Shu, H., Anderson, R. C., \& Wu, N. N. (2000). Phonetic awareness: Knowledge of orthographyphonology relationships in the character acquisition of Chinese children. Journal of Educational Psychology, 92, 56-62.

Shu, H., Chen, X., Anderson, R. C., Wu, N., \& Xuan, Y. (2003). Properties of school Chinese: Implications for learning to read. Child Development, 74, 27-47.

Shu, H., McBride-Chang, C., Wu, S., \& Liu, H. (2006). Understanding Chinese developmental dyslexia: Morphological awareness as a core cognitive construct. Journal of Educational Psychology, 98, $122-133$.

Siegel, L. S., \& Ryan, E. B. (1988). Development of grammatical-sensitivity, phonological, and shortterm memory skills in normally achieving and learning disabled children. Developmental Psychology, 24, 28-37.

Simmons, F., \& Singleton, C. (2000). The reading comprehension abilities of dyslexic students in higher education. Dyslexia, 6, 178-192.

Snowling, M. J. (2000). Dyslexia (2nd ed.). Malden, MA, USA: Blackwell.

Stevenson, H. W., Stigler, J. W., Lucker, G. W., Hsu, C. C., \& Kitamura, S. (1982). Reading disabilities: The case of Chinese, Japanese, and English. Child Development, 53, 1164-1181.

Swanson, H. L. (1994). The role of working memory and dynamic assessment in the classification of children with learning disabilities. Learning Disabilities Research and Practice, 9, 190-202.

Taylor, I., \& Taylor, M. M. (1995). Writing and literacy in Chinese, Korean and Japanese. Philadelphia, PA: John Benjamins. 
Torgesen, J. K., Rashotte, C. A., Greenstein, J., Houck, G., \& Portes, P. (1987). Academic difficulties of learning disabled children who perform poorly on memory span tasks. In H. L. Swanson (Ed.), Memory and learning disabilities: Advances in learning and behavioral disabilities. Greenwich, CT: JAI Press.

Wagner, R. K., \& Torgesen, J. K. (1987). The nature of phonological processing and its causal role in the acquisition of reading skills. Psychological Bulletin, 101, 192-212.

Wagner, R. K., Torgesen, J. K., Laughon, P., Simmons, K., \& Rashotte, C. A. (1993). Development of young readers' phonological processing abilities. Journal of Educational Psychology, 85, 83-103.

Wijsman, E. M., Peterson, D., Leutenegger, A. L., Thomson, J. B., Goddard, K. A. B., Hsu, L., et al. (2000). Segregation analysis of phenotypic components of learning disabilities. I. Nonword memory and digit span. The American Journal of Human Genetics, 67, 631-646.

Wimmer, H., Mayringer, H., \& Landerl, K. (2000). The double-deficit hypothesis and difficulties in learning to read a regular orthography. Journal of Educational Psychology, 92, 668-680.

Wolf, M., \& Bowers, P. G. (1999). The double-deficit hypothesis for the developmental dyslexias. Journal of Educational Psychology, 91(3), 415-438.

Wolf, M., \& Katzir-Cohen, T. (2001). Reading fluency and its intervention. Scientific Studies of Reading, $5,211-239$.

Zhang, C. F., Zhang, J. H., Chang, S. M., \& Zhou, J. (1998). A study of cognitive profiles of Chinese learners' reading disability. Acta Psychologica Sinica, 30, 50-56.

Zhou, X., Zhuang, J., \& Yu, M. (2002). Phonological activation of disyllabic compound words in the speech production of Chinese. Acta Psychologica Sinica, 34, 242-247. 